\title{
The effect of single-nutrient reformulation initiatives on the nutrient profile of foods: a systematic review
}

\author{
Mathilde Gressier, Alexa Segal, Franco Sassi and Gary Frost \\ Imperial College London, London, United Kingdom
}

\begin{abstract}
In an effort to promote population health, governments and businesses are promoting food reformulation initiatives. In most instances, these initiatives have focused on reducing the amount of individual nutrients such as trans-fatty acids (TFA) or sodium, and generally they have been successful at doing so. However, the impact of single-nutrient reformulation on the broader nutrient profile of foods, and on the overall quality of people's diet, remains unclear. We have undertaken a systematic review of evidence of the impact of single-nutrient reformulation on the overall nutrient profile of foods, covering both peer-reviewed studies and grey literature. The outcome of interest was the nutrient composition of the reformulated food or food category. Only 17 studies reported the effect of initiatives on reformulated products' nutrient profile (44 studies reported on only the targeted nutrient). Results are heterogeneous. Six studies investigated the impact of TFA reduction initiatives, showing different impacts on the reformulated products' fat profile, with saturated fats (SFA) decreasing in parallel in some studies, but increasing in others. Similarly, when sodium was reduced, sugar, SFA and calories changed in different directions in different studies. Six studies assessed the effect of mandatory calorie labelling in restaurants, two leading to a reduction in energy intake, one to an increase in sugar and a decrease in unsaturated fatty acids, suggesting a worsened nutrient profile. Of the companies participating in England's sugar reformulation initiative, only $13 \%$ also reduced total energy. Results highlight the limited research on the effect of reformulation strategies on the overall nutrient composition of foods. Only $28 \%$ of the studies that analyse the impact of policies incentivising single nutrient reformulation report the effect on the foods' complete nutrient profile after reformulation. Over a third of the studies show that reducing the targeted nutrient comes at a cost - namely other nutrients may be increased. To produce the desire health impact, reformulation policies must incentivise improvements of the overall nutrient profile of products.
\end{abstract}

\section{Conflict of Interest}

Acknowledgements: MG is the recipient of an unconditional PhD Studentship grant from Nestec. Nestec has no role in the study design, data collection and analysis, or preparation of the protocol and research paper. 